\section{A FORTRAN IV computer program for partitioning chi-square contingency tables}

\section{TED K. KONNERTH and WILLIAM P. DUNLAP \\ Tulane University, New Orleans, Louisiana 70118}

When the chi-square test of significance for frequency data is found to be significant for larger contingency tables, interpretation of results is often aided by a subsequent decomposition or partitioning of the original table into smaller tables. Chi-square statistics calculated from such smaller tables test more discrete, and usually more precise, null hypotheses. The task of partitioning has been approached by several techniques, most of which involve calculating components of the original chi-square, each on one degree of freedom, that basically partition the original contingency table into a series of 2 by 2 tables. Castellan (1965) has provided a brief overview of most of the mathematical techniques used in the process of partitioning. Bresnahan and Shapiro (1966) have outlined a general formula for the computation of chi-square from independently partitioned tables.

The technique for deciding which cells are to be combined or selected for each subtable for the Bresnahan and Shapiro (1966) solution must insure that the partitions are independent, so that the resulting chi-squares, if summed, will equal the original chisquare value. The program described below has been designed to make use of orthogonal coefficients as the means of instructing the computer as to which cells are to be selected or combined. The use of coefficients is advantageous for several reasons: They provide a means of transmitting much information with few data cards; they are easily devised and flexible enough to allow the researcher to make comparisons that will yield a great amount of independent information; they insure that the subsequent comparisons will be independent; and they will allow the user to employ a higher order factorial decomposition of his contingency table, such as those discussed by Winer (1971, pp. 858-859).

To illustrate the technique of devising orthogonal coefficients, a 4 by 4 contingency table, taken from Bresnahan and Shapiro (1966), Example 3b, will be discussed in terms of coefficients. Dealing first with

Preparation and testing of this program was supported by the Tulane University Computer Center. the row coefficients, the first comparison involved the contrast of Rows 1 and 2 to Rows 3 and 4 . We may represent this comparison by the coefficients $(+1+1-1-1)$. The next independent row comparison involved Row 1 vs. 2, and, since Rows 3 and 4 are not involved, we may express this as $(+1-100)$. The third and final independent row comparison, involved only Row 3 vs. 4 , or $(00+1-1)$. The column coefficients for the Bresnahan and Shapiro (1966) example were identical to the rows and can also be summarized by the coefficients $(+1+1-1-1),(+1-100)$, and $(00+1-1)$. These coefficients are independent since they follow the rules of orthogonality, $\Sigma a_{i}=0$ and $\Sigma a_{i} b_{i}=0$, where $a_{i}$ and $b_{i}$ are sets of coefficients. For the reader who is uncertain as to deriving orthogonal coefficients, see Edwards (1968) or Myers (1972) for more worked examples and further clarification.

The program combines the appropriate frequencies from the original $\mathrm{r}$ by $\mathrm{c}$ table into 2 by 2 tables, each on one degree of freedom. There are as many such 2 by 2 tables as there are degrees of freedom of the original table, $(\mathrm{r}-1) \times(\mathrm{c}-1)$. Output includes the cell totals for each 2 by 2 table, the chi-square value, and a diagram of which cells of the original table were combined for that particular partition.

Computer and Language. The program is written in standard FORTRAN IV and runs on an IBM 7044. The program should run with little modification on any computer with a FORTRAN IV compiler.

Availability. A listing of the program may be obtained at no cost by writing Ted Konnerth, Department of Psychology, Tulane University, New Orleans, Louisiana 70118. Instructions for using the program are included as comment statements at the beginning of the program.

\section{REFERENCES}

Bresnahan, J. L., \& Shapiro, M. M. A general equation and technique for the exact partitioning of chi-square contingency tables. Psychological Bulletin, 1966, 66, 252-262.

Castellan, N, J., JR. On the partitioning of contingency tables. Psychological Bulletin, 1965, 64, 330-338.

EDWARDs, A. L. Experimental design in psychological research (3rd ed.). New York: Holt, Rinehart, \& Winston, 1968. Pp. 142-143.

MYERS, J. L. Fundamentals of experimental design (2nd ed.). Boston: Allyn \& Bacon, 1972. P. 358.

WINER, B. J. Statistical principles in experimental design (2nd ed.). New York: McGraw-Hill, 1971. 